\title{
ARTICLE
}

\section{NLRC4 suppresses IL-17A-mediated neutrophil-dependent host defense through upregulation of IL-18 and induction of necroptosis during Gram-positive pneumonia}

\author{
Sagar Paudel ${ }^{1}$, Laxman Ghimire ${ }^{1}$, Liliang Jin ${ }^{1}$, Pankaj Baral ${ }^{1,3}$, Shanshan Cai ${ }^{1}$ and Samithamby Jeyaseelan ${ }^{1,2}$
}

Gram-positive pathogens, including Staphylococcus aureus, cause necrotizing pneumonia. The central feature of S. aureus pneumonia is toxin-induced necroptosis of immune and resident cells, which impedes host defense. However, the role of the NLRC4 in the lung following S. aureus infection remains elusive. Here, we demonstrate that S. aureus activates the NLRC4 to drive necroptosis and IL-18 production, which impaired IL-17A-dependent neutrophil-mediated host susceptibility. In particular, NIrc4 ${ }^{-1}$ mice exhibit reduced necroptosis, enhanced neutrophil influx into the lungs, decreased bacterial burden, and improved host survival. Loss of NLRC4 signaling in both hematopoietic and non-hematopoietic cells contributes to the host protection against S. aureus pneumonia. Secretion of IL-17A by $\gamma \delta$ T cells is essential for neutrophil recruitment into the lungs of N/rc4 ${ }^{-1-}$ mice following infection. Moreover, treatment of wild-type mice with necroptosis inhibitors or genetic ablation of MLKL and IL-18 improves host defense against $S$. aureus infection, which is associated with increased IL-17A $+\gamma \delta T$ cells and neutrophils. Taken together, these novel findings reveal that S. aureus activates the NLRC4 to dampen IL-17A-dependent neutrophil accumulation through induction of necroptosis and IL-18. Thus, modulating the function of the NLRC4 may be an attractive therapeutic approach for treating $S$. aureus infections.

Mucosal Immunology (2019) 12:247-257; https://doi.org/10.1038/s41385-018-0088-2

\section{INTRODUCTION}

Gram-positive pathogens, including Staphylococcus aureus, are the major cause of healthcare-associated pneumonia, sepsis, and mortality in post-influenza infection patients. ${ }^{1,2}$ In particular, the emergence of methicillin-resistant S. aureus (MRSA) as an endemic dominant strain poses a menacing therapeutic challenge in the United States. ${ }^{3}$ Unlike other hospital-acquired S. aureus strains, MRSA causes a broad spectrum of necrotizing infections of skin and soft tissues, including pneumonia, and these infections are associated with high morbidity and mortality, even in immunocompetent individuals. ${ }^{3,4}$ S. aureus pneumonia is characterized by extensive inflammation and localized necrosis leading to loss of alveolar architecture, hemorrhage, and consolidation of lungs. ${ }^{5}$ Emerging evidence suggests that pathogenesis of $S$. aureus pneumonia is largely mediated by over-activation of host inflammatory signaling cascades, although the precise mechanisms underlying this remain elusive.

Despite its antibiotic resistance, the success of $S$. aureus as a highly invasive pathogen is largely attributed to its arsenal of virulence factors, including cytolytic and pore-forming toxins. ${ }^{6}$ All S. aureus strains produce exotoxins or surface proteins, which have diverse targets in multiple cell types. S. aureus has been shown to activate NLRP3 and NOD2, and to target CD11b and ADAM10. ${ }^{7-10}$ Unlike Gram-negative pathogen-induced infections, mice deficient in innate immune molecules including ADAM10, NLRP3, NOD2,
IFNR-1, and TNFR have improved outcomes in S. aureus (Gram positive) pneumonia. ${ }^{10-14}$ In general, these gene-deficient mice show enhanced bacterial clearance, attenuated pro-inflammatory cytokine production, and decreased lung injury/pathology contributing to increased survival against pulmonary $S$. aureus infection. These observations suggest $S$. aureus may have evolved to utilize host innate immune molecules to dampen immune responses. However, the precise mechanism through which $S$. aureus manipulates the host inflammatory machinery to favor the pathogen is not clear.

Necroptosis is a recently identified, pro-inflammatory mode of cell death regulated by receptor-interacting serine-threonine kinases (RIP1/3) and executed by mixed lineage kinase domainlike protein (MLKL). ${ }^{15,16}$ Recently, toxin-induced rapid loss of macrophages via necroptosis has been suggested as a major mechanism of lung damage in $S$. aureus pneumonia. ${ }^{71}$ Similarly, alveolar macrophages exposed to pore-forming toxins from Serratia marcescens undergo necroptosis, which contributes to the severity of pneumonia through substantial loss of critical immune cells and extensive bystander tissue injury. ${ }^{18}$ Genetic ablation of Rip3 or treatment with inhibitors of necroptosis protects alveolar macrophages, which is associated with reduced bacterial burden and improved pneumonia outcomes in mice. ${ }^{17,18}$ However, the mechanism responsible for the necroptosis-mediated depletion of immune cells that

\footnotetext{
${ }^{1}$ Laboratory of Lung Biology, Department of Pathobiological Sciences and Center for Experimental Infectious Disease Research, School of Veterinary Medicine, Louisiana State University (LSU), Baton Rouge, LA 70803, USA and ${ }^{2}$ Section of Pulmonary and Critical Care, Department of Medicine, LSU Health Sciences Center, New Orleans, LA 70112, USA Correspondence: Samithamby Jeyaseelan (jey@lsu.edu)

${ }^{3}$ Present address: Division of Immunology, Department of Microbiology and Immunobiology, Harvard Medical School, Boston, MA 02115, USA
}

Received: 4 May 2018 Revised: 15 August 2018 Accepted: 4 September 2018

Published online: 2 October 2018 


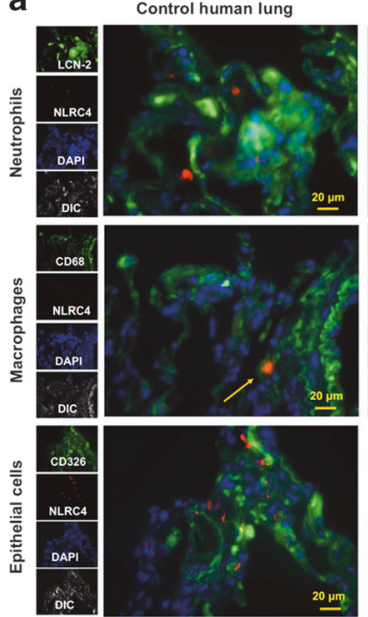

Pneumonic human lung
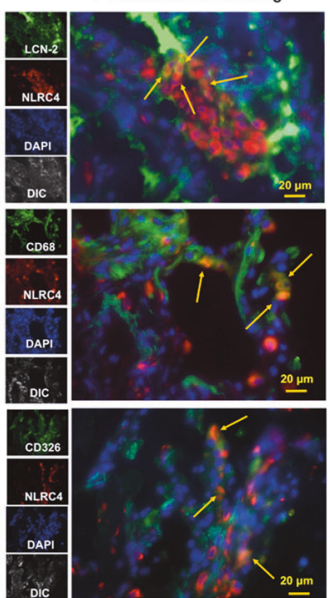

b

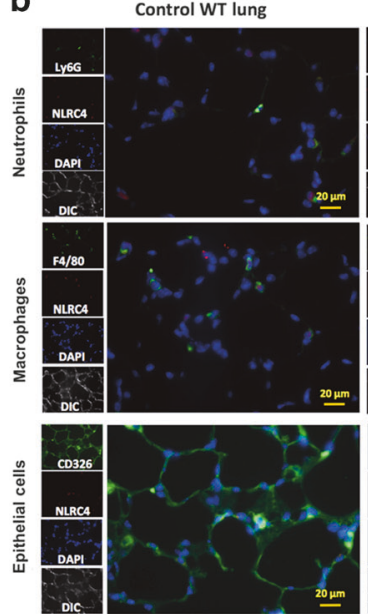

Pneumonic WT lung
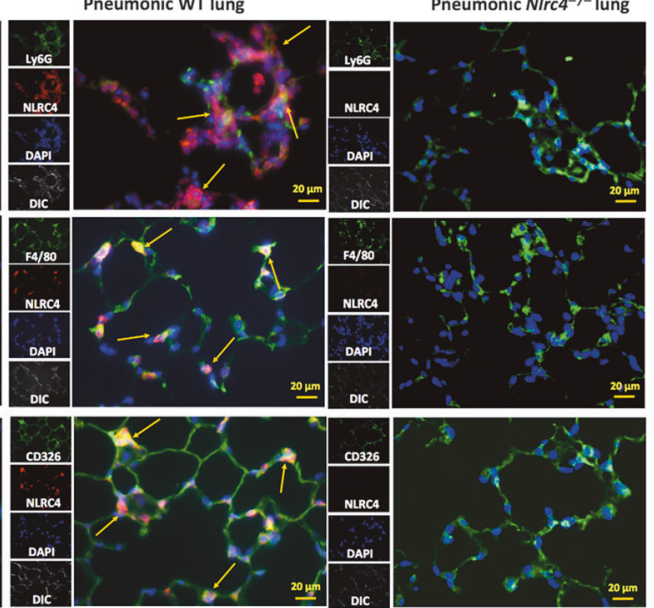

Fig. 1 NLRC4 expression is enhanced in human and murine pneumonic lungs. (a) NLRC4 expression was assessed using immunofluorescence in lung sections from healthy controls and from patients with clinical diagnosis of unspecified bacterial pneumonia. Red staining indicates NLRC4 expression. Neutrophils (Lipocalin- $\left.2^{+}\right)$, macrophages $\left(\mathrm{CD}^{+} 8^{+}\right)$, and epithelial cells $\left(\mathrm{CD} 326^{+}\right)$were stained green. Nuclei were stained with DAPI (blue). Arrowheads (yellow) indicate co-localization of expression of NLRC4 with specific cell markers. Images are representative of five different sections with similar results. (b) NLRC4 expression in lung sections from control and S. aureus-infected WT and Nlrc4 ${ }^{-1-}$ mice at $24 \mathrm{hpi}$. Red staining indicates NLRC4 expression. Neutrophils $\left(\mathrm{Ly}_{6 \mathrm{G}}{ }^{+}\right)$, macrophages $\left(\mathrm{F} 4 / 80^{+}\right)$, and epithelial cells $\left(\mathrm{CD} 326^{+}\right)$are stained green. Nuclei were stained with DAPI (blue). Arrowheads (yellow) indicate co-localization of expression of NLRC4 with specific cell markers. Images are representative of five different sections with similar results. Original magnification, $\times 40$. DAPI, 4',6-diamidino-2-phenylindole; LCN2, lipocalin-2; hpi, hours post infection

impedes bacterial clearance in S. aureus pneumonia largely remains unknown.

Regarding inflammasomes, NLRC4 belongs to the NLR family of proteins containing an N-terminal CARD domain, a central NACHT domain, and a C-terminal leucine-rich repeat domain and is involved in assembly of the inflammasome complex. It was identified as a cytosolic sensor for bacterial flagellin and type III secretion system (T3SS). ${ }^{19,20}$ However, Klebsiella pneumoniae, a non-flagellated bacterium lacking T3SS, and a flagellin-deficient strain of Pseudomonas aeruginosa have been shown to activate the NLRC4, suggesting its activation by other bacterial or endogenous host ligands. ${ }^{21,22}$ Nonetheless, the role of NLRC4 in bacterial pneumonia caused by a Gram-positive organism still remains elusive. ${ }^{21,23}$ Although recent studies linked NLRP3 and ASC to S. aureus toxin-induced necroptosis, the precise mechanism for this has not been elucidated either. ${ }^{7,18}$ Furthermore, $\mathrm{Nlrp}^{-1-}$ mice do not show a completely protective phenotype in $S$. aureus pneumonia, indicating both NLRP3-dependent and independent mechanisms may be important in eliciting lung damage. ${ }^{13}$ To this end, we investigated whether the activation of the NLRC4 is important in the pathogenesis of $S$. aureus pneumonia in a mouse model. We found that NLRC4-driven necroptosis and interleukin-18 (IL-18) suppress IL-17A signaling from $\gamma \delta T$ cells leading to attenuated neutrophil recruitment, which is necessary to eradicate $S$. aureus from the lung. Thus, these findings provide novel insight into how necroptosis and IL18 dampens host defense in $S$. aureus pneumonia and suggests the inhibition of NLRC4 as a potential therapeutic approach for control of $S$. aureus infection in the lungs.

\section{RESULTS}

NLRC4 expression is enhanced in human and murine pneumonic lungs

To determine whether NLRC4 expression is upregulated in human pneumonic lungs, we performed immunofluorescence staining of NLRC4 in lung sections from pneumonic patients and healthy controls. As virulence factors from $S$. aureus are known to target both myeloid and non-myeloid cells, ${ }^{8,10}$ we assessed NLRC4 expression in myeloid cells (neutrophils, macrophages) and nonmyeloid cells (epithelial cells) and found increased expression of NLRC4 in Lipocalin-2 ${ }^{+}$neutrophils, $\mathrm{CD}^{+} 8^{+}$macrophages, and CD326 ${ }^{+}$epithelial cells (Fig. 1a). To explore if NLRC4 expression is increased during Gram-positive pneumonia in mouse lungs, we performed immunofluorescence staining in lung sections at $24 \mathrm{~h}$ post-S. aureus infection. We observed enhanced expression of NLRC4 in $\mathrm{Ly} 6 \mathrm{G}^{+}$neutrophils, $\mathrm{F} 4 / 80^{+}$macrophages, and $\mathrm{CD} 326^{+}$ epithelial cells in infected wild-type (WT) mouse lungs compared to that in control lungs (Fig. 1b). Collectively, our results indicate that both myeloid and non-myeloid cells show upregulation of NLRC4 during bacterial pneumonia.

NLRC4 deficiency contributes to host protection against $S$. aureus pneumonia

To characterize the importance of NLRC4 in S. aureus pneumonia, WT and NIrC4 ${ }^{-1-}$ mice were infected intratracheally (i.t.) with a lethal inoculum of $S$. aureus $\left(2 \times 10^{8} \mathrm{CFU} /\right.$ mouse) and survival was monitored up to $100 \mathrm{~h}$. Compared to WT counterparts, NIrC4 ${ }^{-1-}$ mice showed enhanced survival (Fig. 2a). To determine if the enhanced survival in N/rC4 ${ }^{-1-}$ mice was due to increased bacterial clearance, WT and NIrc4 ${ }^{-1-}$ mice were infected i.t. with a sub-lethal inoculum of $S$. aureus $\left(5 \times 10^{7}\right.$ CFU/mouse). Compared to WT mice at 12 and $24 \mathrm{~h}$ post infection (hpi), NIrc4 ${ }^{-1-}$ mice showed a diminished bacterial burden in the lung and bronchoalveolar lavage fluid (BALF) along with decreased dissemination in the spleen (Fig. 2b-d). We next determined whether the enhanced bacterial clearance was due to augmented neutrophil accumulation in the lungs. Our results revealed that $\mathrm{N} / \mathrm{rC4}^{-1-}$ mice had increased numbers of total white blood cells, neutrophil, and macrophage recruited to the lungs (Fig. 2e-g). Furthermore, unlike N/rc4 ${ }^{-1-}$ mice, WT mice had increased protein leakage, an indicator of lung permeability (Fig. 2h). To assess if the detrimental role of NLRC4 activation is MRSA strain-specific, we infected WT and N/rc4 ${ }^{-1-}$ mice with a methicillin-susceptible $S$. aureus (MSSA) Newman strain. The protective phenotype seen in N/rc4 $4^{-1-}$ mice infected with $S$. aureus was also seen in pneumonia caused by the MSSA strain (Fig. 2i, j). 
a
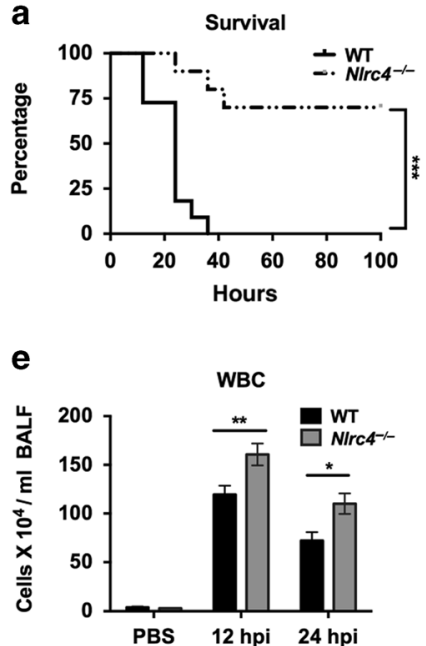

i MSSA_cell influx

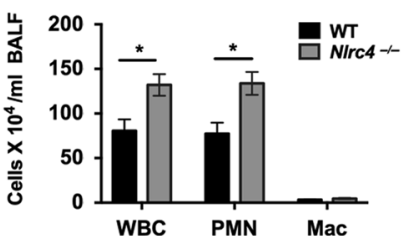

b

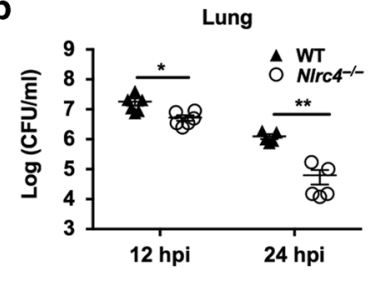

f

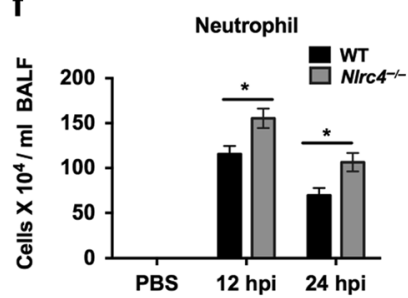

j

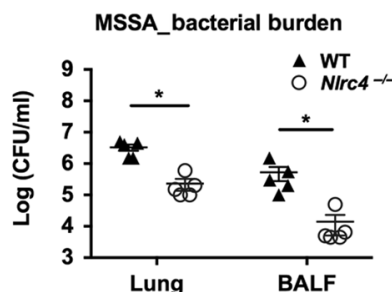

C

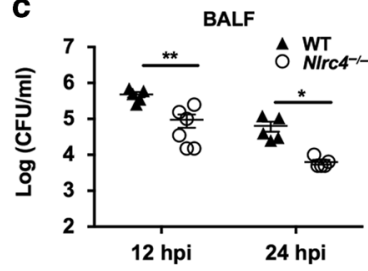

g

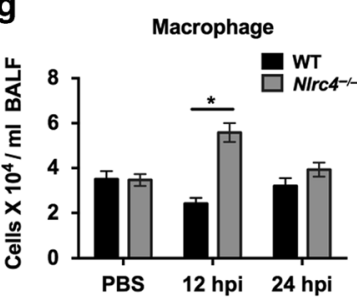

k

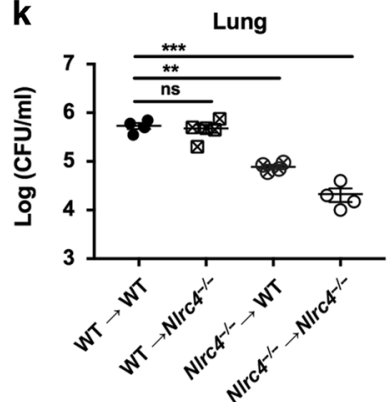

d

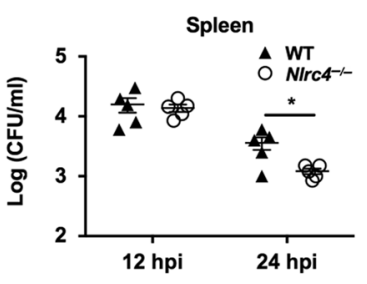

$\mathbf{h}$

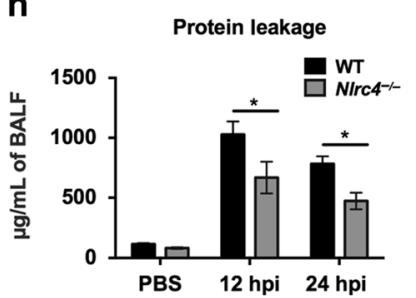

I

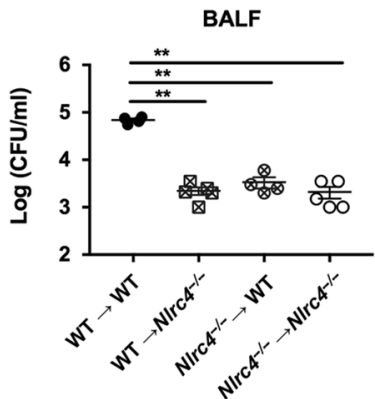

Fig. 2 NLRC4 deficiency confers host resistance against S. aureus pneumonia. (a) WT (control) and Nlrc4 ${ }^{-1-}$ mice were inoculated intratracheally with $2 \times 10^{8} \mathrm{CFU}$ of $\mathrm{S}$. aureus and survival was monitored up to $100 \mathrm{~h}$. A Kaplan-Meier plot is used to show survival of mice from each group. ( $n=10$ mice/group). (b-h) WT and N/rc4 ${ }^{-1-}$ mice were inoculated intratracheally with $5 \times 10^{7}$ CFU of S. aureus or PBS (control). BALF and organs were harvested at the designated time points. Control mice (PBS treated) were sacrificed at $24 \mathrm{hpi}$. The bacterial burden in the (b) lungs, (c) BALF, and (d) spleen was quantitated at 12 and 24 hpi.. Number of (e) total white blood cells, (f) neutrophils, (g) macrophages, and (h) level of total protein were quantified in BALF at 12 and $24 \mathrm{hpi}(n=5-6 \mathrm{mice} /$ pneumonia group, $n=3 \mathrm{mice} / \mathrm{control}$ group). (i, j) WT and NIrc4 ${ }^{-1-}$ mice were inoculated intratracheally with $5 \times 10^{7}$ CFU of S. aureus Newman strain (MSSA). (i) Cellular influx in BALF and (j) bacterial burden in lungs and BALF were determined at $24 \mathrm{hpi}$. (k, I) Bone marrow reconstituted mice were inoculated intratracheally with $5 \times 10^{7} \mathrm{CFU}$ of S. aureus. The bacterial burden in (k) lung and (I) BALF was quantitated at 24 hpi. Each symbol represents a single mouse $(\mathbf{b}-\mathbf{d}, \mathbf{j}-\mathbf{l})$. Data from a representative experiment are shown. All experiments were performed three times, but survival and chimera study were performed twice. Statistical significance was determined by log-rank (a), Mann-Whitney (b-d, j), unpaired $t$-test (e-i), one-way ANOVA (followed by Bonferroni's post hoc comparisons) (k, I). ${ }^{*} p<0.05$; ${ }^{* *} p<0.01$; ${ }^{* *} p<0.001$. BALF, bronchoalveolar lavage fluid; CFU, colonyforming unit; WBC, white blood cell; nd, non-detectable

S. aureus targets both myeloid and non-myeloid cells. ${ }^{7,10,11}$ In Fig. 1a, b, we demonstrate increased expression of NLRC4 in both hematopoietic (myeloid cells) and non-hematopoietic (epithelial cells) cells in human and murine pneumonic lungs. Therefore, we next investigated the contribution of hematopoietic versus non-hematopoietic cells to host protection using bone marrow chimeras. In this context, lethally irradiated WT or NIrC4 ${ }^{-1-}$ mice were reconstituted with RBC-lysed bone marrow cells $\left(4 \times 10^{6} /\right.$ mouse) from donor WT or NIrc4 ${ }^{-1-}$ mice. Two months after reconstitution, chimeric mice were infected with $S$. aureus. Interestingly, the N/rC4${ }^{-1-} \rightarrow \mathrm{NIrC4}^{-1-}, \mathrm{NlrC4}^{-1-} \rightarrow$ WT but not WT $\rightarrow$ Nlrc4 $^{-1-}$ chimeric groups had decreased bacterial burden in lungs as compared with the WT $\rightarrow$ WT group (Fig. 2k). However, the NIrC4 ${ }^{-1-} \rightarrow \mathrm{NIrC4}^{-1-}, \mathrm{NIrC4}^{-1-} \rightarrow \mathrm{WT}$, and $\mathrm{WT} \rightarrow \mathrm{Nlrc}^{-1-}$ groups had reduced bacterial burden in BALF as compared to the WT $\rightarrow$ WT group (Fig. 2i). Taken together, our results suggest NLRC4 activation, in both hematopoietic and non-hematopoietic compartments, is detrimental, and their deficiency in mice displays robust protective phenotype in $S$. aureus pneumonia.
S. aureus activates NLRC4 through PKC- $\delta$ to induce IL-1 $\beta$, IL-18, and necroptosis

S. aureus a-hemolysin has been shown to activate NLPR3 in human and mouse monocytes. ${ }^{7}$ Along the same line, to examine the virulence factors associated with NLRC4 activation, we used an isogenic a-hemolysin-deficient $S$. aureus mutant strain, in human and murine cells. Direct evidence of increased NLRC4 expression in humans was obtained using lung lysates from pneumonic patients and comparing them to lysates from healthy individuals (Supplementary Figure 1a). In a similar manner, S. aureus activated NLRC4 in both human monocyte-like (THP-1) and neutrophil-like (HL-60) cells (Supplementary Figure 1a). To further characterize the role of NLRC4 in response to S. aureus infection, we used THP1 and THP1-NLRC4 cells (stably overexpressing NLRC4). Compared to THP-1 cells, THP1-NLRC4 produced greater amounts of IL-1 $\beta$ and IL-18 in response to $S$. aureus (Fig. 3a, b). In addition, THP1NLRC 4 cells showed increased caspase- 1 activity, as evidenced by their marked staining with the fluorescently labeled inhibitor of caspases reagent (Fig. $3 c$ and Supplementary Figure 1b). We also observed increased expression and phosphorylation of NLRC4 in a 

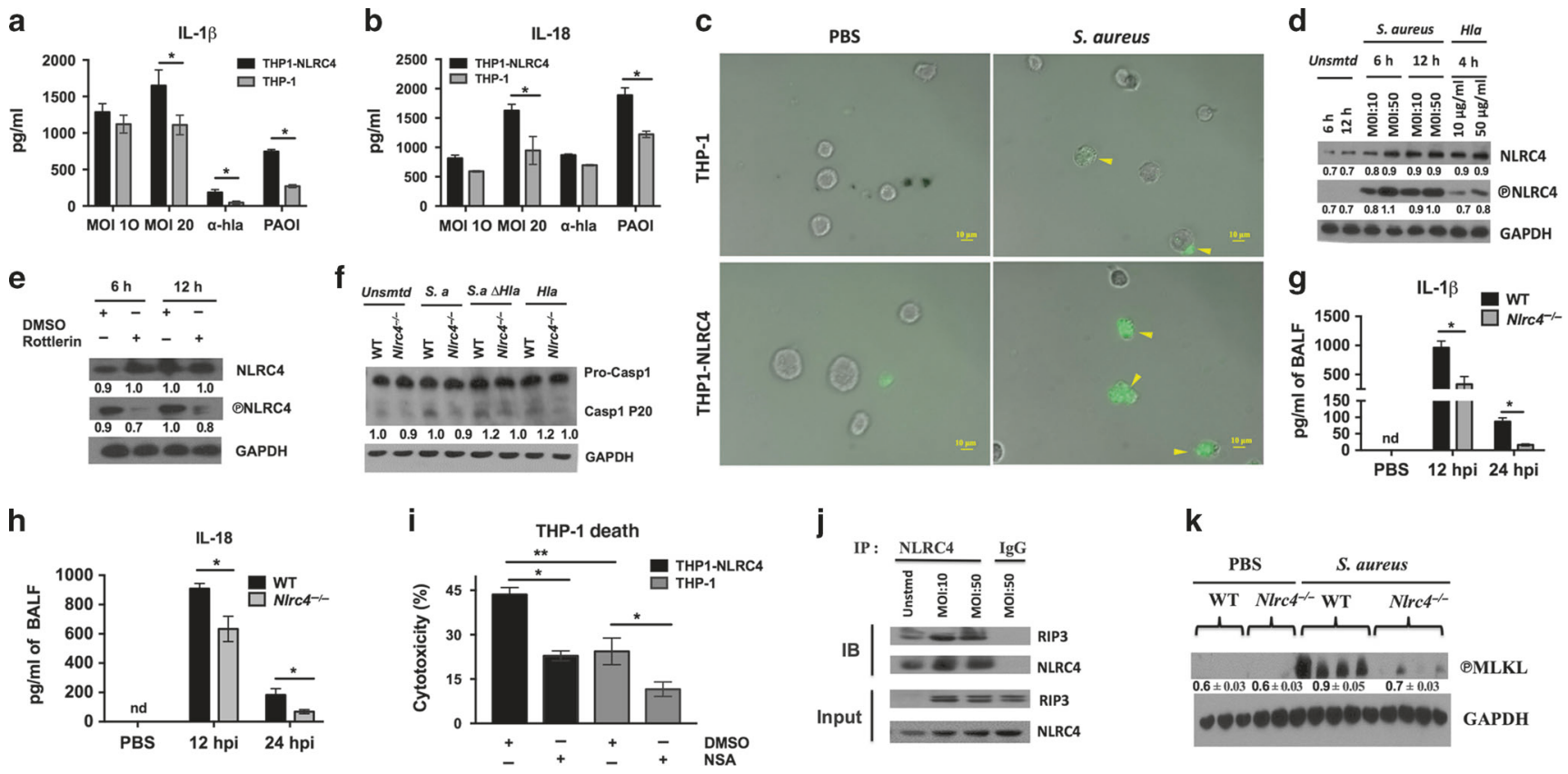

$\mathbf{K}$

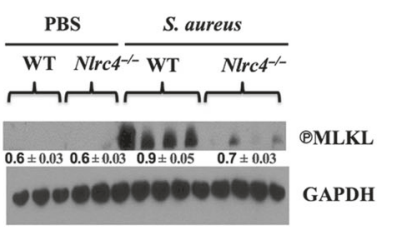

Fig. 3 S. aureus activates NLRC4 through PKC- $\delta$ to induce IL-1 $\beta$, IL-18, and necroptosis. Levels of (a) IL-1 $\beta$ and (b) IL-18 in the supernatant of THP-1 and THP1-NLRC4 cells incubated with S. aureus (MOI 10 or 20$)$, purified $\alpha$-hemolysin ( $25 \mu \mathrm{g} / \mathrm{ml}$ ), or Pseudomonas aeruginosa PAO1 (MOI 10) for $8 \mathrm{~h}$. (c) Active FLICA-caspase-1 (green) was detected in THP-1 and THP1-NLRC4 cells infected with S. aureus (MOI 50) or PBS for $6 \mathrm{~h}$. Original magnification, $\times 40$. (d) Immunoblot analysis of total NLRC4 and phospho-NLRC4 in cell lysates from WT-BMDMs infected with S. aureus (MOI 10 or 50 ) for 6 or $12 \mathrm{~h}$ or stimulated with commercially purified $\alpha$-hemolysin (10 or $50 \mu \mathrm{g} / \mathrm{ml}$ ) for $4 \mathrm{~h}$. (e) Immunoblot analysis of total NLRC4 and phospho-NLRC4 in cell lysates from WT-BMDMs infected with S. aureus (MOI 10) for 6 or $12 \mathrm{~h}$ with or without 1 -h pretreatment with $10 \mu \mathrm{M}$ Rottlerin (PKC- $\delta$ inhibitor). (f) Immunoblot analysis of pro-caspase- 1 and cleaved caspase-1 (p-20) in cell lysates from WT and NIrc4 ${ }^{-1-}$-BMDMs infected with MOI 10 of S. aureus or its isogenic $\alpha$-hemolysin-deficient mutant or stimulated with purified $\alpha$-hemolysin $(50 \mu \mathrm{g} / \mathrm{ml})$ for $6 \mathrm{~h}$. (g, h) WT and N/rc4 ${ }^{-1-}$ mice were inoculated with S. aureus $\left(5 \times 10^{7} \mathrm{CFU} / \mathrm{mouse}\right)$. Level of (g) IL-1 $\beta$ and (h) IL-18 measured BALF collected at 12 and $24 \mathrm{hpi}(n=4-6$ mice/pneumonia group, $n=3$ mice/control group). (i) Cytotoxicity in THP-1 and THP1-NLRC4 cells stimulated with S. aureus (MOI 100) for $2 \mathrm{~h}$ with or without $1 \mathrm{~h}$ pre-treatment with $100 \mu \mathrm{M}$ of necrosulfonamide (NSA). (j) BMDMs from WT mice were left untreated or were incubated with S. aureus (MOI 10 or 50) for $6 \mathrm{~h}$. Cell lysates were immunoprecipitated with ant-NLRC4 Ab or IgG, and then immunoblotted for RIP3 and NLRC4. Whole-cell lysates are shown as the input. (k) Immunoblot analysis of phospho-MLKL in the lung homogenates of WT and NIrc $4^{-1-}$ mice infected with S. aureus $\left(5 \times 10^{7} \mathrm{CFU} / \mathrm{mouse}\right)$. Data from a representative experiment are shown. All experiments were performed three times. In vitro experiments have at least four biological replicates. Numeric values in immunoblot indicate ratio of mean gray scale value of protein of interest to its GAPDH control. Statistical significance was determined by unpaired $t$-test in all experiment except in cell death assay (i), by one-way ANOVA (followed by Bonferroni's post hoc comparisons). ${ }^{*} p<0.05 ;{ }^{* *} p<0.01$. Hla, $\alpha$ hemolysin; $\triangle H$ la, $\alpha$-hemolysin-deficient; MOI, multiplicity of infection; DMSO, dimethyl sulfoxide; IP, immunoprecipitation; IB, immunoblotting

dose- and time-dependent manner with $S$. aureus and purified a-hemolysin in bone marrow-derived macrophages (BMDMs) from WT mice (Fig. 3d and Supplementary Figure 1C). Recently, protein kinase $C-\delta$ (PKC- $\delta$ ) mediated phosphorylation of NLRC4 was shown to be critical for inflammasome activation. ${ }^{24}$ As anticipated, S. aureus-induced NLRC4 phosphorylation was dependent on PKC$\delta$ in macrophages, as Rottlerin (PKC- $\delta$ inhibitor) reduced expression of phospho-NLRC4 (Fig. 3e). We also tested whether S. aureusinduced NLRC4 activation leads to caspase- 1 processing and IL-1 $\beta$ production, key features of inflammasome activation. To this end, we stimulated BMDMs from WT and NIrc4 ${ }^{-1-}$ mice with S. aureus, its isogenic a- hemolysin-deficient mutant, or purified a-hemolysin toxin. As expected, the cleavage of pro-caspase- 1 to its active (phospho-20) form was reduced in N/rC4 ${ }^{-1-}$ BMDMs, which was independent of the expression of a- hemolysin by $S$. aureus (Fig. 3f). This observation indicates $S$. aureus may expresses virulence factors other than a-hemolysin that may serve as activators of NLRC4-dependent caspase- 1 processing. In addition, NLRC4 deficiency modestly reduced IL- $1 \beta$ production in S. aureusinfected BMDMs, so as the pre-treatment with Rottlerin (Supplementary Figure $1 \mathrm{~d}$, e). Furthermore, NIrc4 ${ }^{-1-}$ mice had significant reduction in key inflammasome-dependent cytokines, such as IL-1 $\beta$ and IL-18 (Fig. 3g, h).

S. aureus has been shown to induce necroptosis in lungs. ${ }^{17}$ Therefore, in order to assess the role of NLRC4 in context of necroptosis, we performed cytotoxicity assay in THP-1 and BMDM following S. aureus infection. We detected increased cytotoxicity of THP1-NLRC4 and WT-BMDM compared to THP-1 and NIrC4 ${ }^{-1-}$ BMDMs (Fig. $3 \mathrm{i}$ and Supplementary Figure 1f), providing direct evidence of the involvement of NLRC4 in S. aureus-induced cell death. In addition, pre-treatment with necrostatin-1 (Nec-1: RIP1 inhibitor) or necrosulfonamide (MLKL inhibitor) protected THP1NLRC4 and WT-BMDM from S. aureus-induced necroptosis respectively (Fig. $3 \mathrm{i}$ and Supplementary Figure 1f). Furthermore, we observed increased co-localization of RIP3 and phospho-MLKL in S. aureus-infected WT-BMDMs compared to NIrc4 ${ }^{-1-}$-BMDMs (Supplementary Figures $1 \mathrm{~g}, \mathrm{~h}$ ) as well as the coimmunoprecipitation of RIP3 and NLRC4, indicating their potential interaction in the necrosome complex following $S$. aureus infection (Fig. 3j). Given that MLKL pore formation is a critical step in necroptosis, we sought to detect the level of phospho-MLKL in $S$. aureus-infected lungs. Corroborating our in vitro results, $\mathrm{NIrC4}^{-1-}$ mice show reduced MLKL phosphorylation in their lungs compared to WT controls (Fig. 3k). In a similar manner, WT mice displayed enhanced LDH release and secretion of necroptosisrelated alarmins, such as HMGB-1 and IL-1 $a$, in BALF at 12 and $24 \mathrm{hpi}$ (Supplementary Figures $1 \mathrm{i}-\mathrm{k}$ ). Taken together, our results indicate that $S$. aureus phosphorylates NLRC4 in PKC- $\delta$-dependent manner and activation of which leads to production of IL-1 $1 \beta$, IL-18, and necroptotic cell death. 

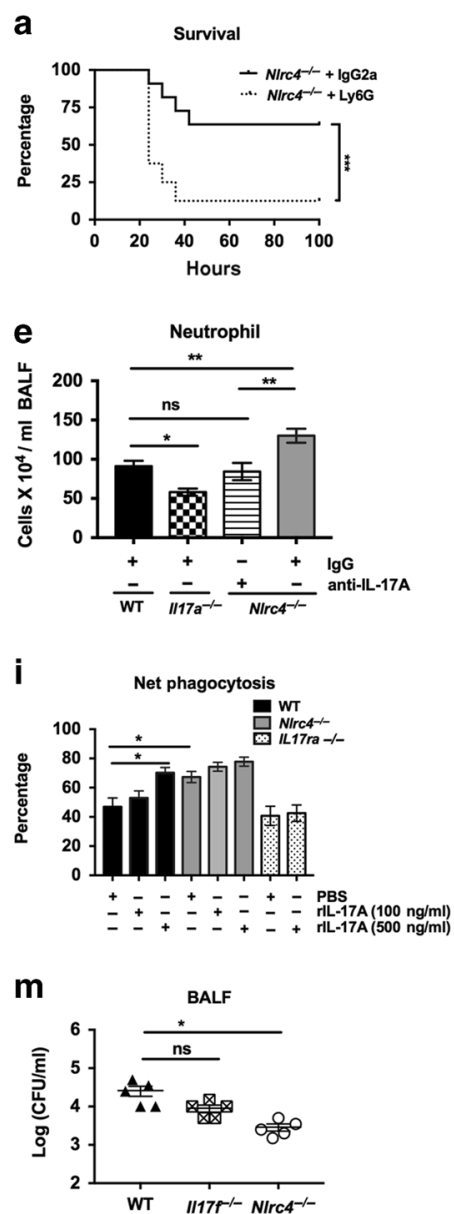
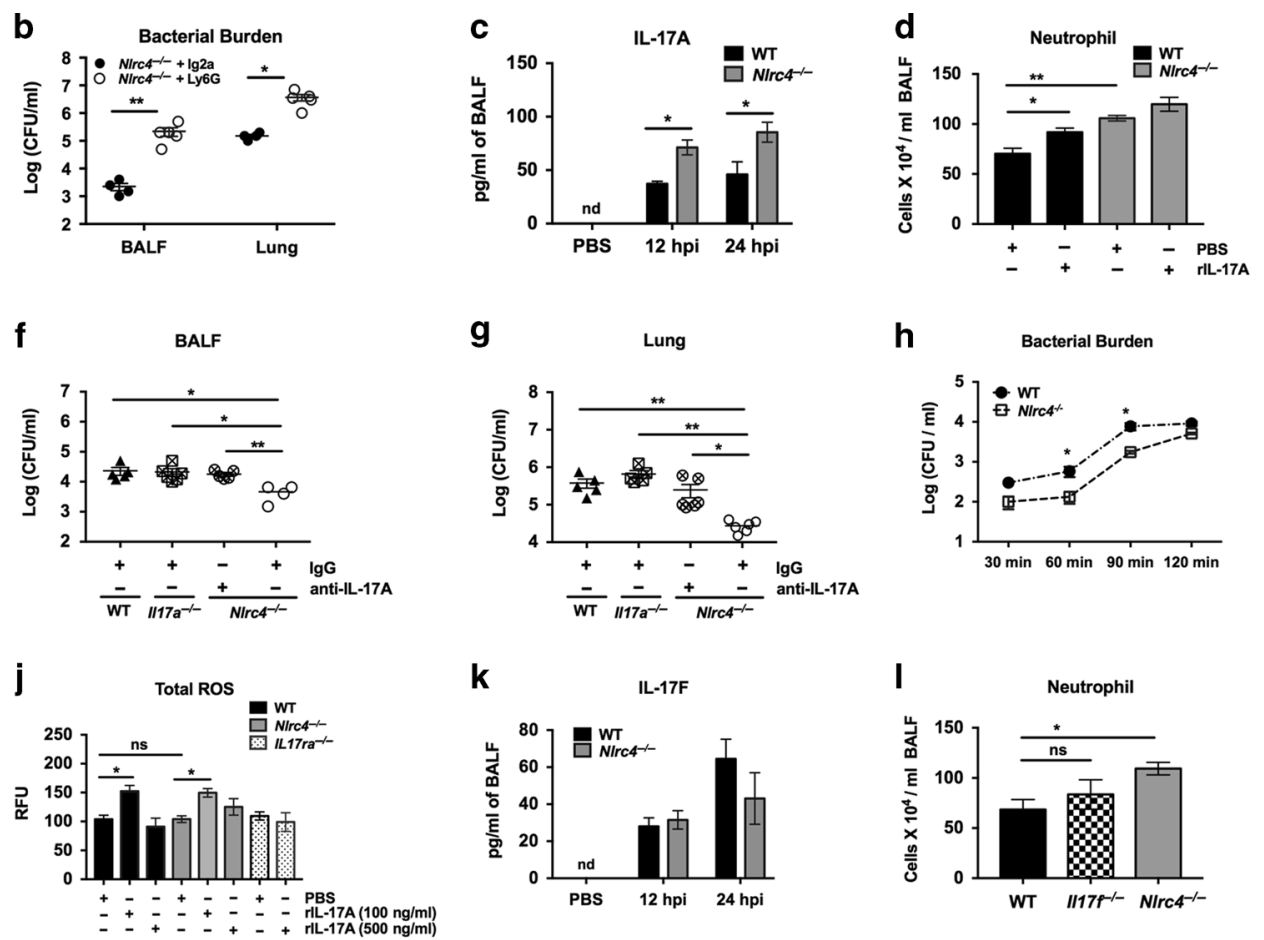

Fig. 4 NLRC4 deficiency promotes IL-17A-mediated neutrophil recruitment and bacterial clearance. (a, b) N/rc4 ${ }^{-/-}$mice were injected with anti-Ly6G (IA8) Ab intraperitoneally at 24 and $2 \mathrm{~h}$ prior to intratracheal infection with $2 \times 10^{8}$ CFU for survival and $5 \times 10^{7}$ CFU for pneumonia. (a) A Kaplan-Meier plot is used to show survival of mice from each group. ( $n=10$ mice/group). (b) Bacterial burden was determined at 24 hpi. Each symbol represents a single mouse. (c,d) WT and N/rc4 ${ }^{-1-}$ mice were inoculated intratracheally with $5 \times 10^{7}$ CFU of S. aureus or PBS followed by administration of recombinant murine IL-17A or PBS $1 \mathrm{~h}$ later. (c) Level of IL-17A at 12 and 24 hpi and (d) number of neutrophils at $24 \mathrm{hpi}$ in the BALF were enumerated. (e-g) WT, II-17a ${ }^{-1-}$ and NIrc4 ${ }^{-1-}$ mice were inoculated intratracheally with $5 \times 10^{7}$ CFU of S. aureus or PBS. $\mathrm{N}_{\mathrm{r} C 4^{-1-}}$ mice received a single dose of anti-IL17A Ab or IgG at $12 \mathrm{~h}$ prior to infection. (e) Number of neutrophils and (f) bacterial burden in BALF and (g) lungs were determined at $24 \mathrm{hpi}$. Each symbol represents a single mouse $(n=4-6 \mathrm{mice} / \mathrm{pneumonia}$ group, $n=3 \mathrm{mice} / \mathrm{control}$

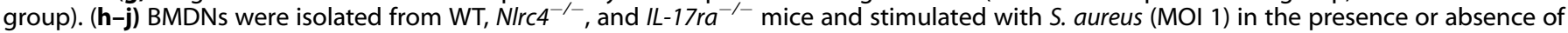
recombinant murine IL-17A (100 or $500 \mathrm{ng} / \mathrm{ml}$ ) or PBS. (h) Intracellular killing of neutrophils was determined at indicated time points by assessing the intracellular bacterial burden as described in Supplementary methods. (i) Net phagocytosis and (j) total ROS production at 1 hpi was determined as described in Supplementary Methods. (k-n) Wild type, $1 /-17 f^{-1-}$, and N/rc $4^{-1-}$ mice were inoculated intratracheally with $5 \times$ $10^{7} \mathrm{CFU}$ of S. aureus. BALF and lungs were collected at 12 and 24 hpi. (k) Level of IL-17F, (I) number of neutrophils in BALF, and bacterial burden in ( $\mathbf{m})$ BALF and (n) lung were determined at $24 \mathrm{hpi}$. Each symbol represents a single mouse $(n=5 \mathrm{mice} / \mathrm{pneumonia}$ group, $n=3$ mice/control group). Data from a representative experiment are shown. All experiments were performed three times. In vitro experiments have at least four biological replicates. Statistical significance was determined by log-rank (a), Man-Whittney (b, h), unpaired $t$-test (c, $\mathbf{k})$, and one-way ANOVA (followed by Bonferroni's post hoc comparisons) (d-g, i, j, I-n). ${ }^{*} p<0.05 ;{ }^{* *} p<0.01$. IgG, immunoglobulin G; ROS, reactive oxygen species

NLRC4 deficiency promotes IL-17A-mediated neutrophil recruitment and bacterial clearance

Neutrophil recruitment is a critical event for bacterial clearance during S. aureus infection. ${ }^{25} \mathrm{~N}_{\mathrm{rc}} 4^{-/-}$mice displayed enhanced neutrophil recruitment (Fig. 2f). Furthermore, neutrophil depletion with anti-Ly6G antibody (IA8) increased the mortality and bacterial burden in N/rC4${ }^{-1-}$ mice (Fig. 4a, b). However, lower levels of pro-inflammatory cytokines in BALF of $\mathrm{NlrC4}^{-1-}$ mice did not explain the higher neutrophil counts, indicating these cytokines (TNF-a, MCP-1, II-6) may not be associated with neutrophil recruitment in $S$. aureus infection (Supplementary Figures S2a-c). It should be noted that IL-17A has been shown to be critical for neutrophil influx and bacterial clearance at the site of $S$. aureus infection. ${ }^{26}$ Thus, we measured the level of IL-17A in BALF and found that IL-17A was increased in N/rc4 ${ }^{-/-}$mice at 12 and $24 \mathrm{hpi}$ (Fig. 4c). To further implicate IL-17A-mediated neutrophil recruitment in N/rc4 ${ }^{-1-}$ mice, administration of recombinant IL-17A in WT mice one-hpi increased neutrophil influx to a level comparable to NIrc4 ${ }^{-/-}$mice (Fig. 4d). Further, ablation of $1117 \mathrm{a}$ or blockade with anti-IL-17A antibody in N/rc4 ${ }^{-1-}$ mice abrogated neutrophil influx, which was associated with an increased bacterial burden in BALF and lungs (Fig. $4 \mathrm{e}-\mathrm{g}$ ). Since IL$17 \mathrm{~A}$ is not directly involved in neutrophil recruitment, we explored whether enhanced recruitment may be due to increased level of CXC chemokines and neutrophil mobilization through IL17A/G-CSF axis. Accordingly, Nlrc4 ${ }^{-/-}$mice also showed increased production of CXCL5, but not CXCL1 and CXCL2, at 12 hpi and higher trend of G-CSF at both 12 and 24 hpi (Supplementary Figures S2d-g). To examine if NLRC4 deficiency also alters the phagocytic function of neutrophils, we utilized a phagocytosis 

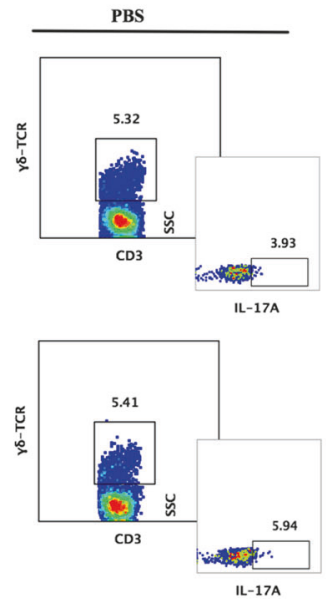

d

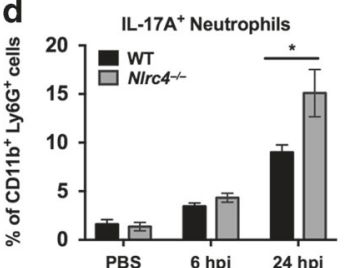

h

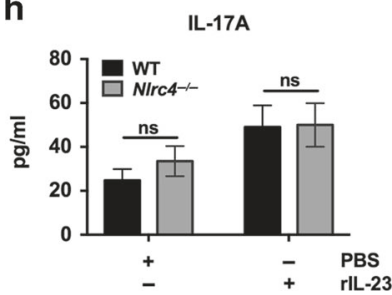

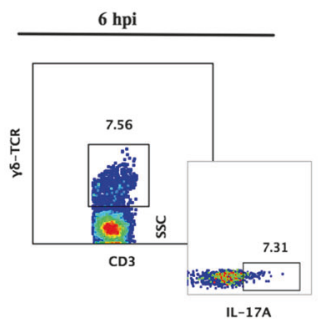
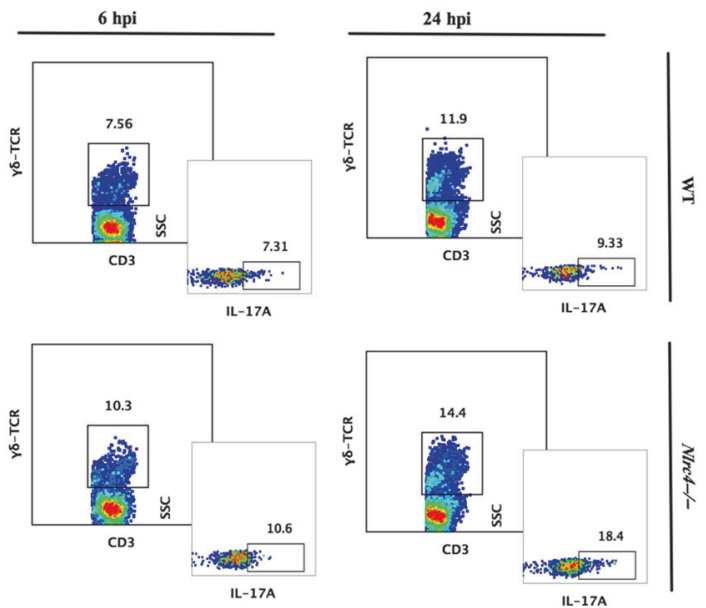

e
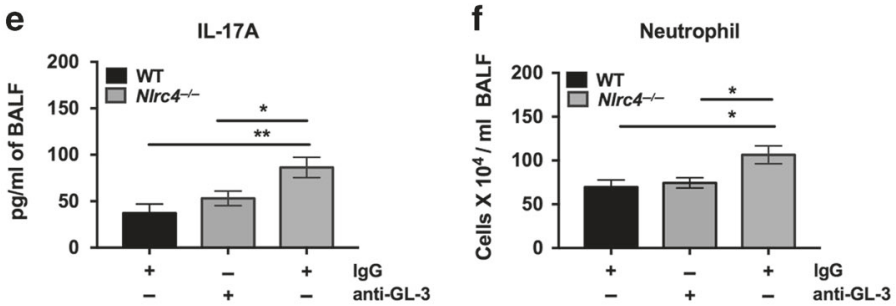

i

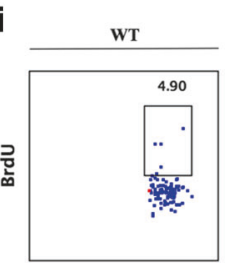

Y $\delta$-TCR

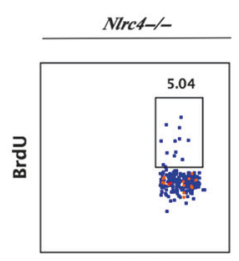

Y $\delta-T C R$ b

$\gamma \delta$ T cells
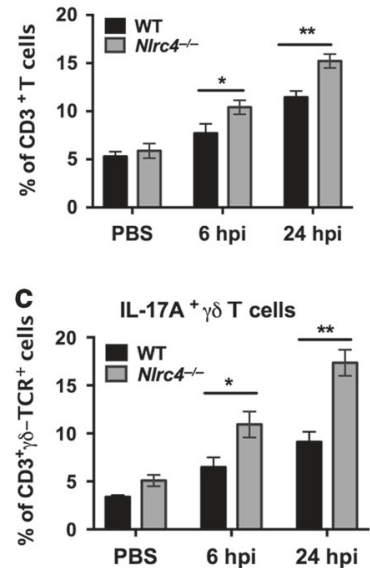

g BALF

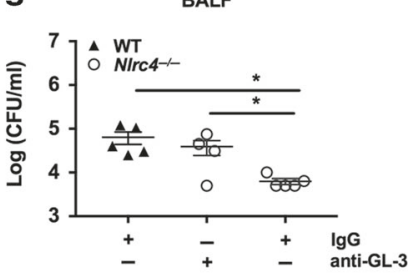

Fig. 5 NLRC4 deficiency augments IL-17A ${ }^{+} \gamma \delta$ T cells and neutrophils to regulate neutrophil influx. (a-d) Flow cytometric analysis of the lungs from WT and NIrc4 ${ }^{-1-}$ mice either uninfected or intratracheally infected with $5 \times 10^{7}$ CFU of S. aureus. (a) FACS plots of CD3 ${ }^{+} \gamma \delta-\mathrm{TCR}^{+}$cells (larger) and IL-17A ${ }^{+} \mathrm{CD}^{+} \gamma \delta-\mathrm{TCR}^{+}$cells (smaller) at 6 and $24 \mathrm{hpi}$. Mice receiving PBS were sacrificed at $24-\mathrm{h}$ time points. (b) The percentage of total $\gamma \delta$ T cells, (c) IL-17A-producing $\gamma \delta$ T cells, and (d) IL-17A producing CD $11 b^{+}$Ly6G-1 ${ }^{+}$neutrophils enumerated by flow cytometry in lungs at 6 and $24 \mathrm{hpi}\left(n=5 \mathrm{mice} /\right.$ pneumonia group, $n=3 \mathrm{mice} / \mathrm{control}$ group). (e-g) WT and N/rc4 ${ }^{-1-}$ mice were inoculated intratracheally with $5 \times$ $10^{7}$ CFU of S. aureus. N/rc4 ${ }^{-1-}$ mice received an intraperitoneal injection of anti- $\gamma \delta-T C R$ ab (GL-3) or lgG at $12 \mathrm{~h}$ prior to infection. (e) Level of IL17A, (f) number of neutrophils, and (g) bacterial burden in BALF was determined at $24 \mathrm{hpi}\left(n=5\right.$ mice/group). (h) The CD3 ${ }^{+} \gamma \delta-\mathrm{TCR}^{+}$cells were sorted from the spleen of the uninfected wild-type and NIrc4 $4^{-1-}$ mice and incubated with S. aureus (MOI 10) for $18 \mathrm{~h}$ in the presence or absence of IL-23 $(40 \mathrm{ng} / \mathrm{ml})$. (h) IL-17A was measured in the supernatant. (i, j) WT and N/rc4 ${ }^{-1-}$ mice were inoculated intratracheally with $5 \times$ $10^{7}$ CFU of S. aureus or PBS. These mice received a single injection of BrdU (1 mg/mouse) intraperitoneally 1-h prior to infection. (i) FACS plot showing $\mathrm{BrdU}^{+} \mathrm{CD}^{+} \gamma \delta-\mathrm{TCR}^{+}$cells and (j) their percentage in the lungs of mice $18 \mathrm{hpi}(n=5 \mathrm{mice} /$ pneumonia group, $n=3 \mathrm{mice} / \mathrm{control}$ group). Data from a representative experiment are shown. All experiments were performed three times. In vitro experiments have at least four biological replicates. Statistical significance was determined by unpaired $t$-test $(\mathbf{b}-\mathbf{d})$ and one-way ANOVA (followed by Bonferroni's post hoc comparisons) $(\mathbf{e}-\mathbf{g}){ }^{*} p<0.05 ;{ }^{* *} p<0.01$. BrdU, bromodeoxyuridine

assay in bone marrow-derived neutrophils (BMDNs). To our surprise, WT-BMDNs show higher intracellular bacterial survival than N/rc4 $4^{-1-}$ - BMDNs following S. aureus infection (Fig. 4h). Further, $\mathrm{NIrC4}^{-1-}$ - BMDNs were also effective at particle uptake (Fig. 4i), suggesting NLRC4 deficiency improved both uptake and clearance in BMDN. Furthermore, the defect in uptake/phagocytosis of WT-BMDN was rescued by pre-treatment with IL-17A, which was associated with increased production of intracellular reactive oxygen species (Fig. $4 \mathrm{i}$, j). Unlike in BMDNs, NLRC4 was dispensable for the phagocytic function of BMDMs (Supplementary Figure S3a). Moreover, deficiency of IL-17F, another IL-17 homodimeric cytokine complex that binds to the same receptors (IL-17RA or IL-17RC), ${ }^{27}$ did not alter neutrophil recruitment or bacterial clearance in $S$. aureus pneumonia (Fig. 4k-n). Collectively, our data indicate that the increased level of IL-17A drives the influx and improves the phagocytic abilities of recruited neutrophil in $\mathrm{Nlrc4}^{-1-}$ mice.

NLRC4 deficiency augments IL-17A ${ }^{+} \gamma \delta \mathrm{T}$ cells and neutrophils to regulate neutrophil influx

Although IL-17A is known as a signature cytokine for $\mathrm{CD}^{+} \mathrm{T}$ cells (Th17 subsets), emerging evidence has revealed that multiple cell types, such as $\gamma \delta \mathrm{T}$ cells, NK/NKT cells, neutrophils, innate lymphoid cells, $\mathrm{CD}^{+} \mathrm{T}$ cells, and colonic epithelial cells, also secrete IL-17A. ${ }^{27}$ To investigate the primary source of IL-17A during the early stage of infection, we utilized intracellular flow cytometry on lung single cell suspensions at 6 and $24 \mathrm{hpi}$. There was an increase in total and IL-17A producing $\mathrm{CD}^{+} \mathrm{\gamma} \delta-\mathrm{TCR}^{+}$cells in the NIrc4 ${ }^{-1-}$ mice at 6 and $24 \mathrm{hpi}$ (Fig. 5a-c). While neutrophils

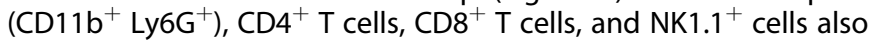


produce IL-17A during S. aureus infection, but NLRC4 deficiency did not alter their number in our model (Supplementary Figures S4a-d). However, IL-17A-producing neutrophils were higher at $24 \mathrm{hpi}$ in $\mathrm{Nlrc4}^{-1-}$ mice, indicating recruited neutrophils help sustain IL-17A levels and recruit more inflammatory cells (Fig. 5d and Supplementary Figure 4a). Human neutrophils activated with $S$. aureus also produced IL-17A (Supplementary Figure 4e). To further implicate $\gamma \delta$ T cells in IL-17A-mediated antimicrobial signaling in N/rc4 ${ }^{-1-}$ mice, we depleted $\gamma \delta \mathrm{T}$ cells using an anti- $\gamma \delta-T C R$ antibody (GL-3). Administration of the GL-3 antibody reduced the level of IL-17A in $\mathrm{NIrC4}^{-1-}$ mice to a level comparable with WT mice receiving isotype-matched IgG control $\mathrm{Ab}$ (Fig. 5e). Furthermore, depletion of $\gamma \delta \mathrm{T}$ cells impaired host defense in $\mathrm{NIrC4}^{-1-}$ mice, as there was decreased neutrophil influx and increased bacterial burden in the BALF of these mice at $24 \mathrm{hpi}$ (Fig. 5f, g). We next asked if genetic deficiency of NLRC4 alters the production of IL-17A from $\gamma \delta T$ cells in addition to their recruitment to the lungs. To this end, we FACS sorted $\mathrm{CD}^{+} \gamma \delta$ $\mathrm{TCR}^{+}$cells from spleens of naïve mice and stimulated them with $S$. aureus in the presence or absence of IL-23 in vitro. Surprisingly, the $\gamma \delta$ T cells from WT and NIrc4 ${ }^{-1-}$ mice did not show altered production of IL-17A following IL-23 stimulation (Fig. 5h). To examine the possibility of increased $\gamma \delta \mathrm{T}$ cell proliferation in lungs of $\mathrm{NlrC}^{-1-}$ mice, we injected BrdU intraperitoneally before $S$. aureus infection and stained for BrdU-positive $\gamma \delta \mathrm{T}$ cells. We found that NLRC4 deficiency did not regulate the proliferation of this cell type in the lungs (Fig. 5i, j). Thus, these data indicate increased frequency of $\gamma \delta T$ cells from early stage and neutrophil at late stage in NIrC4 ${ }^{-1-}$ mice produce IL-17A that is critical of neutrophil recruitment to the lungs in $S$. aureus pneumonia.

NLRC4-driven necroptosis and IL-18 suppress IL-17A-dependent neutrophil recruitment by limiting $\gamma \delta T$ cell expansion

We showed that $S$. aureus-induced NLRC4 activation results in the production of IL-1 $\beta, \mathrm{IL}-18$, and necroptosis in macrophages and lungs (Fig. 3 and Supplementary Fig 1). Necroptosis-mediated loss of immune cells in WT mice, but not in the Rip3 $3^{-1-}$ mice, has been shown to worsen the outcome of S. aureus pneumonia. ${ }^{17}$ Here, we sought to elucidate whether NLRC4-coupled necroptosis suppress IL-17A-mediated antibacterial defense in pneumonia. To confirm necroptosis in vivo, we administered $\mathrm{Nec}-1$ intraperitoneally to block RIP1 and found that it inhibited LDH release in BALF of WT mice at $24 \mathrm{hpi}$ (Fig. 6a). To identify if necroptosis alters $\gamma \delta \mathrm{T}$ cellderived IL-17A signaling axis, we enumerated percentage of total and IL-17A producing $\gamma \delta$ T cells in the lungs of $S$. aureus-infected $\mathrm{MlkI}^{-1-}$ mice. As anticipated, $M / \mathrm{kl}^{-1-}$ mice had a higher percentage of IL-17A ${ }^{+} \gamma \delta$ T cells, but not total $\gamma \delta$ T cells, at 24 hpi (Fig. 6b, c, and Supplementary Figure S5a). Furthermore, the genetic ablation or blockade of MLKL with GW806742X impaired IL-17A production, neutrophil recruitment, and bacterial clearance from BALF and lungs of NIrc4 ${ }^{-1-}$ mice (Fig. $6 \mathrm{~d}-\mathrm{g}$ ). Previous reports have shown that NLRC4 processed IL-18 is deleterious in $P$. aeruginosa-induced pneumonia. ${ }^{23,28}$ In this regard, mice lacking expression of $/ 118$ had increased total and IL-17A-producing $\gamma \delta$ $\mathrm{T}$ cells compared to WT controls, with numbers comparable to those of $\mathrm{Nlrc}^{-1-}$ mice (Fig. 6h, i, and Supplementary Figure 5b). Similarly, administration of recombinant IL-18 in N/rc4 ${ }^{-1-}$ mice decreased both total and IL-17A producing $\gamma \delta T$ cells in the lungs at $24 \mathrm{hpi}$ (Fig. 6h, i, and Supplementary Figure 5b). However, deficiency of IL-1 $\beta$, a key NLRC4-dependent cytokine, did not alter the frequency of $\gamma \delta$ T cells in S. aureus pneumonia (Fig. $6 \mathrm{j}, \mathrm{k}$, and Supplementary Figure 5c). Intriguingly, IL-1 $\beta^{-1-}$ mice did augment host defense with enhanced neutrophil recruitment and bacterial clearance, similar to NIrc4 ${ }^{-1-}$ and $I L-18^{-/-}$(Figures 8I, m). Taken together, our results indicate that NLRC4-mediated necroptosis and IL-18 act synergistically, independent of IL-1 $\beta$, to suppress the recruitment of $\gamma \delta T$ cells, which dampens IL-17A-mediated antibacterial defense in $S$. aureus pneumonia.
Blockade of NLRC4 signaling or necroptosis improves host defense in S. aureus pneumonia

Like other inflammasomes, activation of NLRC4 results in cleavage of caspase- 1 leading to production of IL-1 $\beta$ and IL-18 during $S$. aureus infection (Fig. 3). To determine if deficiency of caspase-1 (downstream of NLRC4) results in a similar outcome in S. aureus pneumonia, mice lacking expression of Casp1/11 or pre-treated with Ac-yvad-cmk (caspase-1 inhibitor) were infected with $S$. aureus. As compared to WT controls, mice lacking Casp1/11 or WT mice pre-treated with Ac-yvad-cmk demonstrate reduced bacterial burden in the BALF and lungs as well as improved survival (Supplementary Figures S6a-C). To determine if blockade of necrosome components also had a similar effect in $S$. aureus pneumonia, we infected mice pre-treated with $\mathrm{Nec}-1$ with $S$. aureus. As anticipated, blockade of RIP1 with Nec-1 enhanced neutrophil recruitment and subsequent bacterial clearance in the BALF, which was associated with improved host survival rates (Supplementary Figures S6d-f). As $S$. aureus-induced NLRC4 activation was dependent on PKC- $\delta$ (Fig. 3e), we investigated the role of PKC- $\delta$ inhibition in host defense. To this end, mice were pre-treated with Rottlerin or dimethyl sulfoxide (DMSO) at $12 \mathrm{~h}$ prior to infection. As expected, Rottlerin pre-treated mice show reduced IL-1 $\beta$ production, increased neutrophil influx, and bacterial clearance at $24 \mathrm{hpi}$ (Supplementary Figures S6g-i). Collectively, our results indicate that the inhibition of the NLRC4 signaling or necroptosis signaling cascades improves host defense against $S$. aureus infection.

\section{DISCUSSION}

The pathogenesis of Gram-positive (S. aureus) pneumonia is mediated by its several virulence factors, such as exotoxins and surface proteins. These virulence factors target different innate signaling molecules in infiltrating leukocytes and resident epithelial cells. Moreover, growing evidence indicates that $S$. aureus is able to exploit innate signaling pathways, as mice deficient in Adam10, Nlrp3, Nod2, Ifnr-1, and Tnfr expression showed augmented host defense against this pathogen. ${ }^{10-14}$ Although toxin-induced necroptotic lung damage has been implicated in the pathogenesis of $S$. aureus pneumonia, ${ }^{17}$ the precise mechanism underlying the subversion of host immunity is largely unknown. We report that NLRC4 activation leads to necroptotic cell death and IL-18 production, which in turn impedes host defense in $S$. aureus pneumonia. Furthermore, we demonstrate a novel mechanism by which NLRC4-driven necroptosis and IL-18 production suppress $\gamma \delta \mathrm{T}$ cell-derived IL-17Adependent neutrophil recruitment during $S$. aureus infection. Finally, we show that blockade or genetic deficiency in NLRC4 signaling cascade and necroptosis pathway improve host defense in the context of $S$. aureus pneumonia.

Pulmonary host defense regulated by the NLRC4 is protective against Gram-negative pathogens, such as $K$. pneumoniae, ${ }^{21} L$. pneumophila, ${ }^{29,30}$ and $B$. pseudomallei, ${ }^{31}$ but deleterious against $P$. aeruginosa. $^{23,32}$ In contrast, our study reports NLRC4 activation is deleterious in the context of Gram-positive pathogens, as N/rc4 ${ }^{-/-}$ mice show improved host defense and survival. In accordance with this, activation of other NOD-like receptors has also been shown to be detrimental during $S$. aureus infection. For example, Nod $2^{-/-}$and Nlrp3 $3^{-1-}$ mice are less susceptible to S. aureus pneumonia. ${ }^{12,13}$ Because NLRP3 recognizes a broad range of pathogens, including S. aureus, ${ }^{33}$ NLRP3 activation was thought to be critical for the pathogenesis of $S$. aureus pneumonia. However, $\mathrm{Nlrp3}^{-1-}$ mice do not have a remarkable phenotype in S. aureus pneumonia, suggesting involvement of an NLRP3-independent host defense mechanism in the response to this pathogen. ${ }^{13} \mathrm{We}$ showed both myeloid and resident cells express NLRC4 during $S$. aureus pneumonia. Our bone marrow chimeras reveal that NLRC4 expression in both compartments is detrimental with regard to 


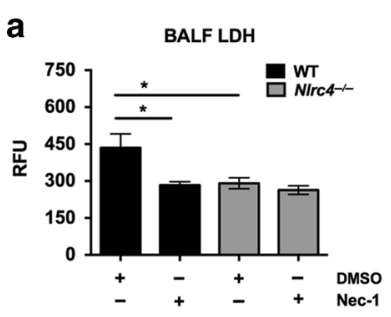

e
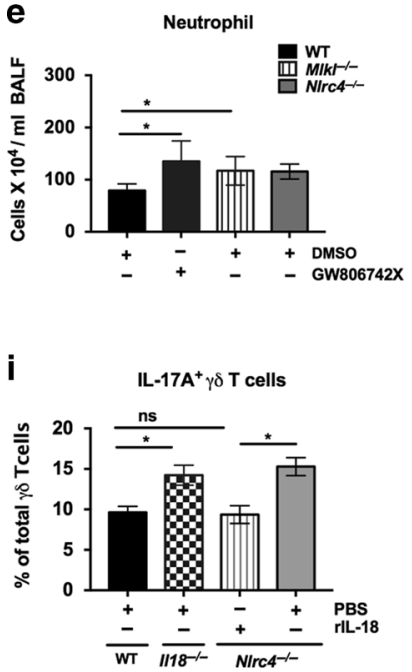

m

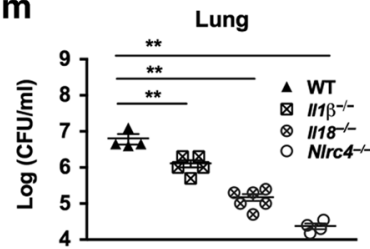

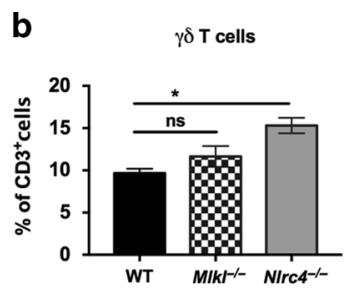

f
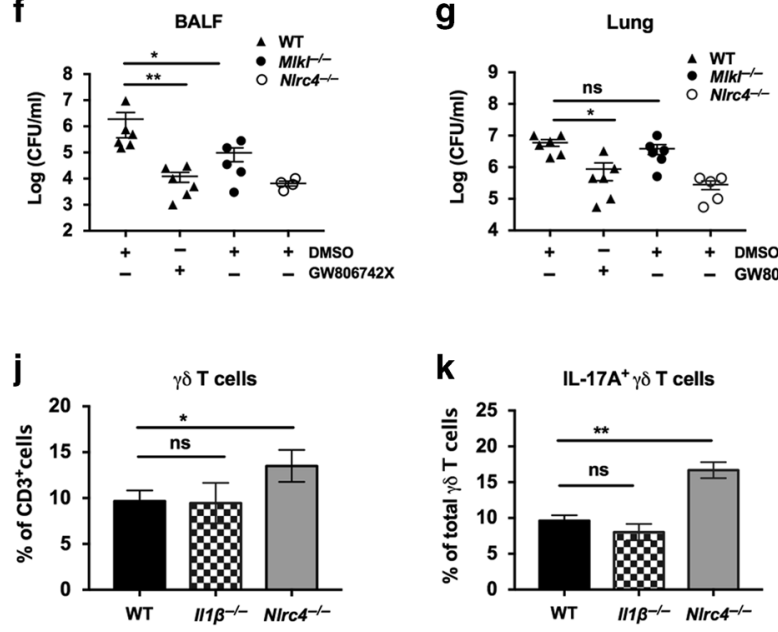

g

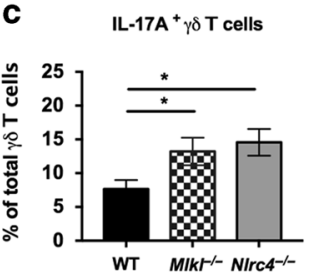

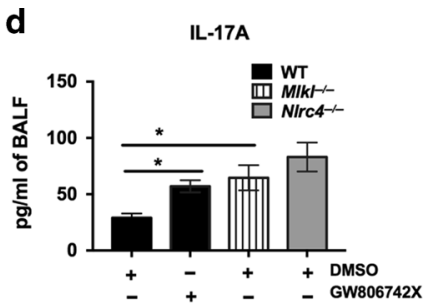

h

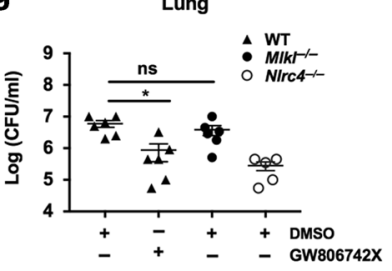

$\gamma \delta$ T cells
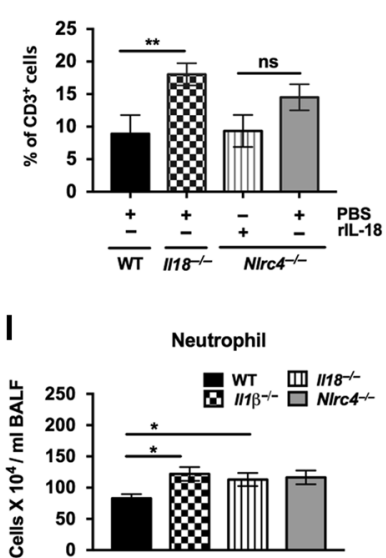

Fig. 6 NLRC4-driven necroptosis and IL-18 suppress 17A-dependent neutrophil recruitment by limiting $\gamma \delta \mathrm{T}$ cell expansion. (a) WT and NIrc $4^{-/-}$mice were treated with Necrostatin-1 or DMSO intraperitoneally $12 \mathrm{~h}$ prior to infection with S. aureus ( $5 \times 10^{7} \mathrm{CFU} / \mathrm{mouse}$ ). RFU of LDH release in BALF was measured at $24 \mathrm{hpi}\left(n=5\right.$ mice /group). (b-g) WT, Mlkl ${ }^{-1-}$, and Nlrc4 ${ }^{-1-}$ mice were infected with S. aureus $\left(5 \times 10^{7} \mathrm{CFU} /\right.$ mouse) intratracheally with administration of GW806742X (MLKL inhibitor) or DMSO intraperitoneally $1 \mathrm{~h}$ prior. BALF and lungs were harvested at $24 \mathrm{hpi}$. (b) The percentage of total $\gamma \delta \mathrm{T}$ cells and (c) IL-17A-producing $\gamma \delta \mathrm{T}$ cells in the lungs were determined by flow cytometry. (d) IL-17A level, (e) number of neutrophils, and (f) bacterial burden in BALF, and (g) lungs were quantitated. Each symbol represents a single mouse ( $n=4-6$ mice/group). (h-m) WT, $I I-18^{-1-}, I I-1 \beta^{-/-}$, and Nlrc4 ${ }^{-1-}$ mice were infected with S. aureus $\left(5 \times 10^{7} \mathrm{CFU} / \mathrm{mouse}\right)$ intratracheally. At $24 \mathrm{hpi}$, the lungs were harvested and processed for flow cytometric analysis. (h) The percentage of total $\gamma \delta$ T cells and (i) IL-17A producing

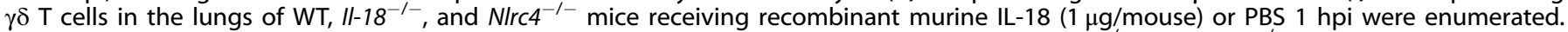
(j) The percentage of total $\gamma \delta$ T cells and (k) IL-17A-producing $\gamma \delta$ T cells in the lungs of WT, $I I-1 \beta^{-I-}$, and NIrc4 $4^{-1-}$ mice. (I) The number of neutrophils in BALF and $(\mathbf{m})$ bacterial burden in the lungs at $24 \mathrm{hpi}$. Each symbol represents a single mouse $(n=4-6 / g r o u p)$. Data from a representative experiment are shown. All experiments were performed three times. Statistical significance was determined by one-way ANOVA (followed by Bonferroni's post hoc comparisons) in all experiments. ${ }^{*} p<0.05 ;{ }^{* *} p<0.01$

bacterial control. It is important to note that the $\mathrm{WT} \rightarrow \mathrm{N} / \mathrm{rC} 4^{-1-}$ group (hematopoietic) had defective bacterial control in the lungs compared to other chimeric groups. Since neutrophil are a major player of S. aureus clearance and $\mathrm{NIrC4}^{-1-}$ neutrophils are more efficient in controlling bacterial burden, it is possible that NLRC4 expression in myeloid cells of the WT $\rightarrow$ NIrc4 $^{-1-}$ (only hematopoietic expression) group may have dampened phagocytic function of these cells.

Based on data from experiments in THP-1 cells and BMDMs, the NLRC4 was important for caspase- 1 activity and IL- $1 \beta$ production in response to $S$. aureus infection. Our findings also indicate that $\alpha-$ hemolysin induces phosphorylation of the NLRC4, which is a PKC$\delta$-dependent process like in $S$. typhimurium-induced NLRC4 activation in macrophages. ${ }^{24}$ However, a-hemolysin expression in S. aureus was dispensable for caspase- 1 processing. In addition, pulmonary infection with $K$. pneumoniae or $P$. aeruginosa (nonflagellated strains) has been shown to activate the NLRC4 in vivo, ${ }^{21,22}$ indicating that virulence factors other than flagellin can activate NLRC4. In this regard, recent reports related to the involvement of NLRC4 activation in obesity-associated tumors, in progression of melanoma tumors, and in diabetic nephropathy suggest that endogenous host proteins may serve as signals for NLRC4 activation. ${ }^{34-36}$ Therefore, it is possible that unidentified bacterial components of $S$. aureus or host-derived proteins may act as homologs to known NLRC4-activating bacterial signals.

A number of $S$. aureus toxins are known to activate necroptosis, a highly pro-inflammatory mode of cell death. S. aureus-derived pore-forming toxins like Hla, Leukocidin $A B$, and PSMs leading to cytotoxicity has been well characterized. ${ }^{7,8,37}$ Further, necroptosis results in irreparable loss of alveolar macrophages and 
anti-inflammatory signals, impeding clearance of S. aureus. ${ }^{17}$ In addition to reduced phosphorylation of MLKL in the lungs of Nirc4 ${ }^{-1-}$ mice, we also found that NLRC4 interacts with RIP3, suggesting a potential role of NLRC4 in necrosome formation. However, it remains unknown if NLRC4 activation also alters the $\mathrm{K}^{+}$ efflux or contributes to membrane destabilization leading to cell lysis. Unlike $\mathrm{Nlrp}^{-/-}$, Nod $2^{-/-}$, or Rip ${ }^{-/-}$mice infected with $\mathrm{S}$. aureus, ${ }^{12,13,17}$ the central feature of protection in $\mathrm{NlrC4}^{-1-}$ mice is their ability to recruit sufficient numbers of neutrophils to the lungs, which is impaired in WT mice. In N/rC4 ${ }^{-1-}$ mice, this occurs through the increased frequency of $\gamma \delta$ T cells, which provide the IL17A signal to drive neutrophil influx. Previous studies have shown that IL-17A stimulates granulopoesis in bone marrow and regulates expression of CXC chemokines in lungs to recruit neutrophils. ${ }^{38,39}$ In our model, it appears that IL-17A may have contributed to neutrophil recruitment via increased CXCL5 and G-CSF production in N/rC4 ${ }^{-1-}$ mice. While numerous immune cells can produce IL$17 \mathrm{~A}$ at different stages of infection, many studies have highlighted the importance of early IL-17A production by $\gamma \delta \mathrm{T}$ cells during infection with S. aureus ${ }^{26,40,41}$ and other extracellular bacteria. ${ }^{39,42}$ In addition to $\gamma \delta$ T cells, the increased frequency of IL-17A + neutrophils at $24 \mathrm{~h}$ post infection may explain that neutrophils are also involved in the recruitment through an autocrine loop. Interestingly, MLKL deficiency or blockade of necroptosis with Nec1 or MLKL inhibitors improves bacterial clearance, which is also associated with an increased frequency of IL-17A ${ }^{+} \gamma \delta$ T cells and neutrophil recruitment. In addition to necroptosis, NLRC4-induced IL-18 production was found to suppress the $\gamma \delta \mathrm{T}$ cell-IL-17A axis, as IL-18 deficiency resulted in improved neutrophil recruitment and clearance of S. aureus. Similar to our results, IL-18 has also been shown to impede host defense in pneumonia and other disease models. ${ }^{23,28,43}$ However, IL-1 $\beta$, another NLRC4-dependent cytokine, was surprisingly found to be dispensable for this pathway. Thus, these two seemingly disparate NLRC4-dependent mechanisms, the necroptosis and IL-18, work synergistically to impede neutrophil recruitment in S. aureus pneumonia. However, future studies will be required to determine how deficiency of necroptosis and IL-18 leads to the increased frequency of IL-17A-producing $\gamma \delta$ T cells as early as 6 hpi.

The precise mechanism through which PKC- $\delta$-dependent NLRC4 phosphorylation occurs following Gram-positive (S. aureus) infection remains unclear. It should be noted that a wide range of pathogens or host-derived factors, along with environmental pollutants, can activate NLRP3. ${ }^{33}$ Along the same lines, it is possible that NLRC4 may recognize host-derived ligands during bacteria-induced inflammation in addition to recognizing pathogens and/or their components. For example, since $S$. aureus -derived pore-forming toxins destabilize the membrane of cells, it is possible that endogenous inflammatory ligands are released and subsequently activates NLRC4. However, elucidation of the specific ligands and mechanisms responsible for NLRC4 activation during Gram-positive S. aureus infection of the lung will require future studies.

\section{METHODS}

Animals

NIrc4 ${ }^{-1-}$ mice were generated as described. ${ }^{44}$ The generation of $I I-$ $17 a^{-1-},\left\|1-17 f^{-1-},\right\| 1-17 \mathrm{ra}^{-/-}, \operatorname{Casp}^{-1-} / 11^{-/-},\left\|1-1 \beta^{-1-},\right\| 1-18^{-/-}$, and $\mathrm{MlkI}^{-\mathrm{L}^{\prime}}$ were described previously. ${ }^{22,27,45-48}$ All mouse strains were backcrossed onto the $\mathrm{C} 57 \mathrm{BL} / 6 \mathrm{~J}$ genetic background at least 10 times. Age- and gender-matched WT controls were used. The Institutional Animal Care and Use Committee of the Louisiana State University approved all animal experiments.

Pneumonia model

The S. aureus USA300 strain, its isogenic a-hemolysin (hla)deficient, and MSSA Newman strain were used to induce pneumonia, as described previously. ${ }^{21,49}$ Bacterial preparation is detailed in Supplementary Methods. After anesthesia with xylazine/ketamine mix, mice were i.t. inoculated with $S$. aureus at a concentration of $5 \times 10^{7} \mathrm{CFUs} / 50 \mu \mathrm{l} /$ mouse for pneumonia or $2 \times 10^{8} \mathrm{CFUs} / 50 \mu \mathrm{l} / \mathrm{mouse}$ for survival. Neutrophil depletion was achieved with intraperitoneal injection of $500 \mu \mathrm{g}$ of anti-Ly6G ab (IA8) (Biolegend) $24 \mathrm{~h}$ and $2 \mathrm{~h}$ prior to infection. ${ }^{50}$ In some experiments, mice were injected intraperitoneally with $300 \mu \mathrm{g}$ of necrostatin-1 (RIP1 inhibitor) (Calbiochem), $150 \mu \mathrm{g}$ of Ac-yvad-cmk (Caspase-1 inhibitor) (Caymen Chemical), $200 \mu \mathrm{g}$ of murine anti-IL$17 \mathrm{~A}$ antibody (eBiosccience), $100 \mu \mathrm{g}$ of anti- $\gamma \delta-\mathrm{TCR}$ antibody (Biolegend), or $200 \mu \mathrm{g}$ of Rottlerin (PKC- $\delta$ inhibitor) (Calbiochem) $12 \mathrm{~h}$ prior to infection as described with or without slight modifications. ${ }^{17,51,52}$ In other experiments, $100 \mu \mathrm{l}$ of $10 \mu \mathrm{M}$ of GW806742X (MLKL inhibitor) (Synkinase) was administered intraperitoneally $1 \mathrm{~h}$ prior to infection. ${ }^{18}$ In additional experiments, mice were treated i.t. with $1 \mu \mathrm{g}$ recombinant IL-17A or IL-18 (R\&D Systems) 1 hpi. $^{21,26}$ Control mice received phosphate-buffered saline (PBS) or DMSO, or control rat lgG2a, as appropriate.

BALF collection, cell counts, and bacterial burden

BALF and organs were collected as described earlier. ${ }^{21}$ In brief, mice were sacrificed, the trachea was exposed and cannulated with 20-gauge catheter, and the lungs were flushed four times with $0.8 \mathrm{ml}$ of PBS containing heparin/dextrose. Total and differential cell counts were performed on BALF using light microscopy utilizing Quik-Dip Stain. Lungs/spleen were excised, homogenized in PBS, and plated in serial dilutions on TSA plates to quantitate the bacterial burden in organs after overnight incubation at $37^{\circ} \mathrm{C}$.

Immunofluorescence microscopy

Immunofluorescence staining for NLRC4 expression on tissue slides was performed as described previously. ${ }^{39}$ Paraffinembedded lung sections from healthy or diseased humans (with clinical diagnosis of unspecified bacterial pneumonia) were obtained from Biochain Institute Inc. USA. Following primary antibodies were used: anti-NLRC4 Ab (Cell Signaling), antilipocalin-2 $\mathrm{Ab}$ for PMNs (R\&D systems), anti-CD68 Ab for macrophages (Biolgend), and anti-CD326 Ab for epithelial cells (Biolegend). For mouse slides, we used anti-NLRC4 Ab (EMD Millipore), anti-Ly6G ${ }^{+} \mathrm{Ab}$ for PMNs (Biolegend), anti-CD326 Ab for epithelial cells (Biolegend), and the anti-F4/80 Ab for myeloid cells (Biolegend). Similarly, anti-RIP3 Ab (Cell Signaling) and anti-MLKL (phospho S345) Ab (Abcam) were used to study necroptosis in mouse BMDMs. Alexa-conjugated secondary antibodies (Invitrogen) were used.

\section{Flow cytometry}

For flow cytometric analysis, lungs were excised, minced, digested for $90 \mathrm{~min}$ at $37^{\circ} \mathrm{C}$ in collagenase $(2 \mathrm{mg} / \mathrm{ml}$ ) (Worthington) and DNase I $(20 \mathrm{U} / \mathrm{ml})$ (Roche) to obtain single cell suspensions. The following surface marker antibodies were purchased from eBioscience: anti-CD11b (clone M1/70), Ly6G (1A8), F-4/80 (BM8), CD3 (17A2), CD4 (GK1.5), CD8a (53-6.7), ү $\delta$-TCR (GL3), and NK1.1 (PK136). For intracellular staining, cells were incubated for $4-5 \mathrm{~h}$ with $3 \mathrm{mg} / \mathrm{ml}$ of brefeldin A (eBioscience). The BD Cytofix/ Cytoperm Kit was used to permeabilize and fix cells before staining with IL-17A (17B7, eBioscience). Isotype controls were used for compensation. Cells were acquired on a FACs caliber (BD Biosciences). FlowJo 10 (Treestar) was used to analyze data.

Western blotting and immunoprecipitation

Western blotting for lung homogenates and cell lysates was performed as described in previous publications. ${ }^{21}$ Antibodies to NLRC4 (EMD Millipore), NLRC4 (phospho S533) (ECM Biosciences), Caspase-1 (Adipogen), RIP3 (Cell Signaling), MLKL (phospho S345) (Abcam), and GAPDH (Cell Signaling) were used. The 
immunoprecipitation Kit, Dynabeads ${ }^{\mathrm{TM}}$ Protein G (Invitrogen), was used to immunoprecipitate RIP3 and NLRC4 according to the manufacturer's protocol.

Bone marrow chimeras

Bone marrow transplantation was performed to create $\mathrm{Nlrc4^{-1- }}$ chimera mice wherein the NIrc4 deficiency was confined to either the hematopoietic cells (NIrc4 $4^{-1-} \rightarrow$ WT) or non-hematopoietic tissue (WT $\rightarrow \mathrm{Nlrc}^{-1-}$ ), as described in our previous publications. ${ }^{21,39}$ In brief, recipient mice were lethally irradiated with a single dose of $1000 \mathrm{rad}$. Each recipient mouse received a tail vein injection of marrow cells $\left(4 \times 10^{6} /\right.$ mouse) and was kept on $0.2 \%$ neomycin-sulfate drinking water for 2 weeks. Pneumonia was induced at 2 months after the reconstitution. Four groups were generated (WT $\rightarrow$ WT, WT $\rightarrow \mathrm{NIrC4}^{-1-}, \mathrm{NlrC}^{-1-} \rightarrow \mathrm{WT}$, and NIrC4${ }^{-1}$ $\rightarrow$ Nlrc $4^{-1-}$ ). Our routine procedure results in at least $75-85 \%$ reconstitution of blood leukocytes from donor mice, as confirmed by flow cytometry on donor cells expressing GFP.

Statistical analysis

Data are expressed as means \pm standard error of mean (s.e.m.) unless otherwise stated. Prism 7.0a software (GraphPad Software Inc.) was used for statistical analyses. The unpaired $t$-test, Mann-Whitney $U$ test (non-parametric), or one-way ANOVA (followed by Bonferroni's post hoc comparisons) were used to analyze differences between groups, as appropriate. Survival curves (Kaplan-Meier plot) were compared using log-rank tests. ${ }^{*} p<0.05,{ }^{* *} p<0.01$, and ${ }^{* *} p<0.001$ were considered significant.

\section{ACKNOWLEDGEMENTS}

This work was supported by NIH grants F31HL137287 to S.P., R01Al113720, R01HL091958, and R21Al133681-01 to S.J., and R01HL133336 to S.C. We thank Drs. Juliane Bubeck-Wardenburg (Washington University School of Medicine, St. Louis) for a-hemolysin-deficient strains of S. aureus USA300 and Jiahuai Han (Xiamen University, China) for providing $\mathrm{Mlkl}^{-1-}$ mice. We thank Marilyn Dietrich for flow cytometry. We also thank the Lung Biology Laboratory members, including Tirumalai Rangasamy, Scott Bergeron, Sangeetha Ravi Kumar, Joseph DeCorte, and John Le for helpful discussion.

\section{AUTHOR CONTRIBUTIONS}

Conceived and designed experiments: S.P. and S.J. Performed experiments: S.P., L.G., L.J., P.B. and S.C. Analyzed the data: S.P. and S.J. Wrote the paper: S.P. and S.J.

\section{ADDITIONAL INFORMATION}

The online version of this article (https://doi.org/10.1038/s41385-018-0088-2) contains supplementary material, which is available to authorized users.

Competing interests: The authors declare no competing interests.

\section{REFERENCES}

1. Chertow, D. S. \& Memoli, M. J. Bacterial coinfection in influenza: a grand rounds review. JAMA 309, 275-282 (2013).

2. David, M. Z. \& Daum, R. S. Community-associated methicillin-resistant Staphylococcus aureus: epidemiology and clinical consequences of an emerging epidemic. Clin. Microbiol. Rev. 23, 616-687 (2010).

3. King, M. D. et al. Emergence of community-acquired methicillin-resistant Staphylococcus aureus USA 300 clone as the predominant cause of skin and softtissue infections. Ann. Intern. Med. 144, 309-317 (2006).

4. Francis, J. S. et al. Severe community-onset pneumonia in healthy adults caused by methicillin-resistant Staphylococcus aureus carrying the Panton-Valentine leukocidin genes. Clin. Infect. Dis. 40, 100-107 (2005).

5. Garnier, F. et al. Pneumonia and new methicillin-resistant Staphylococcus aureus clone. Emerg. Infect. Dis. 12, 498-500 (2006).

6. Diep, B. A. \& Otto, M. The role of virulence determinants in community-associated MRSA pathogenesis. Trends Microbiol. 16, 361-369 (2008).

7. Craven, R. R. et al. Staphylococcus aureus alpha-hemolysin activates the NLRP3inflammasome in human and mouse monocytic cells. PLOS ONE 4, e7446 (2009).
8. DuMont, A. L. et al. Staphylococcus aureus LukAB cytotoxin kills human neutrophils by targeting the CD11b subunit of the integrin Mac-1. Proc. Natl. Acad. Sci. USA 110, 10794-10799 (2013).

9. Girardin, S. E. et al. Nod2 is a general sensor of peptidoglycan through muramyl dipeptide (MDP) detection. J. Biol. Chem. 278, 8869-8872 (2003).

10. Inoshima, I. et al. A Staphylococcus aureus pore-forming toxin subverts the activity of ADAM10 to cause lethal infection in mice. Nat. Med. 17, 1310-1314 (2011).

11. Gomez, M. I. et al. Staphylococcus aureus protein A induces airway epithelial inflammatory responses by activating TNFR1. Nat. Med. 10, 842-848 (2004).

12. Kapetanovic, R. et al. Contribution of NOD2 to lung inflammation during Staphylococcus aureus-induced pneumonia. Microbes Infect. 12, 759-767 (2010).

13. Kebaier, C. et al. Staphylococcus aureus alpha-hemolysin mediates virulence in a murine model of severe pneumonia through activation of the NLRP3 inflammasome. J. Infect. Dis. 205, 807-817 (2012).

14. Parker, D., Planet, P. J., Soong, G., Narechania, A. \& Prince, A. Induction of type I interferon signaling determines the relative pathogenicity of Staphylococcus aureus strains. PLoS Pathog. 10, e1003951 (2014).

15. Cai, Z. et al. Plasma membrane translocation of trimerized MLKL protein is required for TNF-induced necroptosis. Nat. Cell Biol. 16, 55-65 (2014).

16. Kaczmarek, A., Vandenabeele, P. \& Krysko, D. V. Necroptosis: the release of damage-associated molecular patterns and its physiological relevance. Immunity 38, 209-223 (2013).

17. Kitur, K. et al. Toxin-induced necroptosis is a major mechanism of Staphylococcus aureus lung damage. PLoS Pathog. 11, e1004820 (2015).

18. Gonzalez-Juarbe, N. et al. Pore-forming toxins induce macrophage necroptosis during acute bacterial pneumonia. PLoS Pathog. 11, e1005337 (2015).

19. Miao, E. A. et al. Cytoplasmic flagellin activates caspase-1 and secretion of interleukin 1 beta via Ipaf. Nat. Immunol. 7, 569-575 (2006).

20. Miao, E. A. et al. Innate immune detection of the type III secretion apparatus through the NLRC4 inflammasome. Proc. Natl. Acad. Sci. USA 107, 3076-3080 (2010).

21. Cai, S., Batra, S., Wakamatsu, N., Pacher, P. \& Jeyaseelan, S. NLRC4 inflammasomemediated production of IL-1 beta modulates mucosal immunity in the lung against gram-negative bacterial infection. J. Immunol. 188, 5623-5635 (2012).

22. Sutterwala, F. S. et al. Immune recognition of Pseudomonas aeruginosa mediated by the IPAF/NLRC4 inflammasome. J. Exp. Med. 204, 3235-3245 (2007).

23. Faure, E. et al. Pseudomonas aeruginosa type-3 secretion system dampens host defense by exploiting the NLRC4-coupled inflammasome. Am. J. Respir. Crit. Care Med. 189, 799-811 (2014).

24. Qu, Y. et al. Phosphorylation of NLRC4 is critical for inflammasome activation. Nature 490, 539-542 (2012).

25. Rigby, K. M. \& DeLeo, F. R. Neutrophils in innate host defense against Staphylococcus aureus infections. Semin. Immunopathol. 34, 237-259 (2012).

26. Cho, J. S. et al. IL-17 is essential for host defense against cutaneous Staphylococcus aureus infection in mice. J. Clin. Invest. 120, 1762-1773 (2010).

27. Iwakura, Y., Ishigame, H., Saijo, S. \& Nakae, S. Functional specialization of interleukin-17 family members. Immunity 34, 149-162 (2011).

28. Schultz, M. J. et al. Interleukin-18 impairs the pulmonary host response to Pseudomonas aeruginosa. Infect. Immun. 71, 1630-1634 (2003).

29. Pereira, M. S. et al. Activation of NLRC4 by flagellated bacteria triggers caspase-1dependent and -independent responses to restrict Legionella pneumophila replication in macrophages and in vivo. J. Immunol. 187, 6447-6455 (2011).

30. Pereira, M. S., Marques, G. G., Dellama, J. E. \& Zamboni, D. S. The Nlrc4 inflammasome contributes to restriction of pulmonary infection by flagellated Legionella spp. that trigger pyroptosis. Front. Microbiol. 2, 33 (2011).

31. Ceballos-Olvera, I., Sahoo, M., Miller, M. A., Del Barrio, L. \& Re, F. Inflammasomedependent pyroptosis and IL-18 protect against Burkholderia pseudomallei lung infection while IL-1beta is deleterious. PLoS Pathog. 7, e1002452 (2011).

32. Cohen, T. S. \& Prince, A. S. Activation of inflammasome signaling mediates pathology of acute P. aeruginosa pneumonia. J. Clin. Invest. 123, 1630-1637 (2013).

33. Ravi Kumar, S. et al. Emerging roles of inflammasomes in acute pneumonia. Am. J. Respir. Crit. Care Med. 197, 160-171 (2018).

34. Kolb, R. et al. Obesity-associated NLRC4 inflammasome activation drives breast cancer progression. Nat. Commun. 7, 13007 (2016).

35. Janowski, A. M. et al. NLRC4 suppresses melanoma tumor progression independently of inflammasome activation. J. Clin. Invest. 126, 3917-3928 (2016).

36. Yuan, F. et al. Involvement of the NLRC4-inflammasome in diabetic nephropathy. PLOS ONE 11, e0164135 (2016).

37. Wang, R. et al. Identification of novel cytolytic peptides as key virulence determinants for community-associated MRSA. Nat. Med. 13, 1510-1514 (2007).

38. Schwarzenberger, P. et al. IL-17 stimulates granulopoiesis in mice: use of an alternate, novel gene therapy-derived method for in vivo evaluation of cytokines. J. Immunol. 161, 6383-6389 (1998).

39. Cai, S., Batra, S., Del Piero, F. \& Jeyaseelan, S. NLRP12 modulates host defense through IL-17A-CXCL1 axis. Mucosal Immunol. 9, 503-514 (2016). 
NLRC4 suppresses IL-17A-mediated neutrophil-dependent host defense... S Paudel et al.

40. Cheng, P. et al. Role of gamma-delta T cells in host response against Staphylococcus aureus-induced pneumonia. BMC Immunol. 13, 38 (2012).

41. Maher, B. M. et al. Nlrp-3-driven interleukin 17 production by gammadeltaT cells controls infection outcomes during Staphylococcus aureus surgical site infection. Infect. Immun. 81, 4478-4489 (2013).

42. Dubin, P. J. \& Kolls, J. K. Th17 cytokines and mucosal immunity. Immunol. Rev. 226, 160-171 (2008).

43. Dolinay, T. et al. Inflammasome-regulated cytokines are critical mediators of acute lung injury. Am. J. Respir. Crit. Care Med. 185, 1225-1234 (2012).

44. Mariathasan, S. et al. Differential activation of the inflammasome by caspase- 1 adaptors ASC and Ipaf. Nature 430, 213-218 (2004).

45. Kuida, K. et al. Altered cytokine export and apoptosis in mice deficient in interleukin-1 beta converting enzyme. Science 267, 2000-2003 (1995).

46. Zheng, $\mathrm{H}$. et al. Resistance to fever induction and impaired acute-phase response in interleukin-1 beta-deficient mice. Immunity 3, 9-19 (1995).
47. Takeda, K. et al. Defective NK cell activity and Th1 response in IL-18-deficient mice. Immunity 8, 383-390 (1998).

48. Ye, P. et al. Requirement of interleukin 17 receptor signaling for lung CXC chemokine and granulocyte colony-stimulating factor expression, neutrophil recruitment, and host defense. J. Exp. Med. 194, 519-527 (2001).

49. Kulkarni, R. et al. Cigarette smoke extract-exposed methicillin-resistant Staphylococcus aureus regulates leukocyte function for pulmonary persistence. Am. J. Respir. Cell Mol. Biol. 55, 586-601 (2016).

50. Ocuin, L. M. et al. Neutrophil IL-10 suppresses peritoneal inflammatory monocytes during polymicrobial sepsis. J. Leukoc. Biol. 89, 423-432 (2011).

51. Skelsey, M. E., Mellon, J. \& Niederkorn, J. Y. Gamma delta T cells are needed for ocular immune privilege and corneal graft survival. J. Immunol. 166, 4327-4333 (2001).

52. Markel, G. et al. The involvement of IL-17A in the murine response to sub-lethal inhalational infection with Francisella tularensis. PLOS ONE 5, e11176 (2010). 